\title{
Acknowledgement to Reviewers of High-Throughput in 2018
}

\author{
High-Throughput Editorial Office \\ MDPI, St. Alban-Anlage 66, 4052 Basel, Switzerland \\ Published: 11 January 2019
}

Rigorous peer-review is the corner-stone of high-quality academic publishing. The editorial team greatly appreciates the reviewers who contributed their knowledge and expertise to the HighThroughput's editorial process over the past 12 months. In 2018, a total of 41 papers were published in the journal, with a median time to first decision of 19 days and a median time to publication of 46 days. The editors would like to express their sincere gratitude to the following reviewers for their cooperation and dedication in 2018:
Alekseyev, Yuriy
Alexander, Rebecca
Alicea, Bradly
Amara, Neri
Bandini, Erika
Bauer, Joshua A.
Berteotti, Chiara
Brewin, Nick
Broszczak, Daniel
Brown, Laurence
Caldarelli, Stefano
Callebaut, Isabelle
Cannataro, Mario
Casals-Terre, Jasmina
Chun, Honggu
Cipak Gasparovic, Ana
Dash, Biraja
Delorme, Vincent
Deng, Peng
Dorado, Pedro
Eboigbodin, Kevin
Elaiw, Ahmed M.
Elsayed, Wael A.
El-Seedi, Hesham R.
El-Sofany, Walaa
Fabris, Linda
Feng, Zhiwei
Gerber, Susanne
Guarascio, Massimo
Hardiman, Gerard
Head, Steven
Hernandez, Wenndy
Hertz, Daniel L.

\author{
Hizel, Candan \\ Holland, Michael \\ Horvath, Dragos \\ Huang, Hsiao-Yun \\ Iadarola, Paolo \\ Ikonomopoulou, Maria P \\ Ilagan, Ma. Xenia G. \\ Ino, Kosuke \\ Islam, Mohammad Mazharul \\ Ito, Takuhiro \\ Ivanov, Alexander $\mathrm{V}$ \\ Jain, Vaibhav \\ Janga, Madhusudhana \\ Jose, Jiney \\ Jurman, Giuseppe \\ Kadimisetty, Karteek \\ Kajdanowicz, Tomasz \\ Kaliviotis, Stathis \\ Kaneko, Kimiyuki \\ Kaplan, Shauna \\ Kim, Seokmin \\ Klein, Matthias S. \\ Komarova, Natalia V. \\ Kostic, Tanja \\ Kukreja, Muskan \\ Kumar, Sunil \\ Kunz, Meik \\ Kuzmin, Victor \\ Larson, Nicholas B. \\ Liby, Karen \\ Ligterink, Wilco \\ Linsen, Lars \\ Lopez-Camarillo, Cesar
}


Mahmood, Khalid

Mao, Meng

Marie-helene, David-Cordonnier

Markiv, Anatoliy

Mason-Suares, Heather

Mathieu, Didier

Mazzotti, Fabio

Meerang, Mayura

Menon, Vipin

Miernyk, Jan A.

Mohanta, Tapan

Morris, Douglas

Nagai, Kouhei

Naryzhny, Stanislav

Nees, Matthias

Neogi, Ujjwal

Novelli, Antonio

Osolodkin, Dmitry

Pal, Chandal

Pandey, Santosh

Papantonis, Argyris

Pekar, Stanislav

Perez Tur, Jordi

Peter, Emanuel K.

Pham, Thang V.

Pislariu, Catalina

Portelius, Erik

Puigvert, Jordi Carreras

Rahmanpour, Rahman

Raikhy, Gaurav

Raj, Nitin

Rajpurohit, Surendra

Ramachandran, Vinoy

Riar, Amanjot Kaur

Roland, Pochet

Rozycki, Bartosz
Ruddraraju, Kasi Viswanatharaju

Sachkova, Maria

Sampathi, Shilpa

Sankaranarayanan, Nehru Viji

Santarelli, Lory

Santoni-Rugiu, Eric

Sengupta, Arjun

Shen, Lishuang

Smyth, Gordon K.

Spakowicz, Dan

Stanberry, Larissa

Stephenson, Richard

Stern, Adi

Sturtevant, Joy

Suarez-Ulloa, Victoria

Sudhir, Putty-Reddy

Sumoy, Lauro

Sun, Fengjie

Sun, Wei

Suzurikawa, Jun

Sztuba-Solinska, Joanna

Tagliaferri, Pierosandro

Thakkar, Shraddha

Thompson, Jeffrey

Tiziani, Stefano

Tullo, Apollonia

Vittorioso, Paola

Vogt, Andreas

Vos, Robin

Wang, Jun

Wang, Junjie

$\mathrm{Xu}$, Suowen

Zaravinos, Apostolos

Zhang, Ruiyong

Zhang, Tianyu

Zhang, Yu

(C) 2019 by the authors. Licensee MDPI, Basel, Switzerland. This article is an open access article distributed under the terms and conditions of the Creative Commons Attribution (CC BY) license (http://creativecommons.org/licenses/by/4.0/). 